\title{
Novel Approach to Unsupervised Mobility Assessment Tests: Field Trial For aTUG
}

\author{
T. Frenken*, M. Lipprandt*, M. Brell*, M. Gövercin ${ }^{\dagger}$, S. Wegel ${ }^{\dagger}$, E. Steinhagen-Thiessen ${ }^{\dagger}$, A. Hein* \\ *OFFIS - Institute for Information Technology, Escherweg 2, D-26121 Oldenburg, Germany, Email: thomas.frenken@offis.de \\ ${ }^{\dagger}$ Geriatrics Research Group, Charité - Universitätsmedizin, Reinickendorferstr. 61, D-13347 Berlin, Germany
}

\begin{abstract}
A novel approach to performing unsupervised mobility assessment tests in domestic environments is presented. As a part of the aTUG concept the approach is based on the idea to segment assessment tests into components made up of recurring movement patterns which are measured independently by use of ambient sensor technologies. Quality criteria are defined which compute a score of eligibility for usage of sensor data to assess a certain test component. Valid component measurements are recombined to complete assessment tests according to a technical assessment test description defining the flow of segments and their constraints. An experiment has been conducted within a field trial with five elderly people aged 64-84 years over five weeks. The flats of all people were equipped with home automation (HA) sensors. A laser range scanner (LRS) was placed in one flat. Results from the fully-equipped flat show that the presented quality criteria are suitable to select LRS measurements according to their eligibility to assess a certain component. HA sensors and the LRS were used to compute a self-selected gait velocity of $0.71 \mathrm{~m} / \mathrm{s}$ unsupervised at home. TUG using the aTUG apparatus and a stopwatch was used as clinical reference data yielding a mean gait velocity of $1.18 \mathrm{~m} / \mathrm{s}$. For the described setting a difference of $0.47 \mathrm{~m} / \mathrm{s}$ between capacity and performance in gait velocity was found.
\end{abstract}

Index Terms-aTUG, Timed Up and Go (TUG), Mobility Assessment, Domestic Assessment, Laser Range Scanner, LIDAR, Force Sensors, Sensor Fusion

\section{INTRODUCTION}

The double aging of the society is a result of the demographic change. It leads to more elderly people requiring health services and less young people financing and providing these services. While the increased need of care in elderly people is also related to their multi-morbidity, the interchangeeffects of the diseases hamper to make an exact diagnosis.

In geriatrics the ultimate aim of a treatment is to recover and maintain an independent lifestyle of patients. Estimating the functional status of patients and compensating or removing deficits by means of rehabilitation or provision of aid is more important than a detailed diagnosis. The estimation of a geriatric patient's functional status happens within the geriatric assessment by use of various standardized assessment tests.

Mobility is a fundamental requirement for an independent lifestyle [1]. Pathological changes in mobility are related to increased need of care and increased risk of falling [2]. Therefore, estimation of mobility is a central aspect of each geriatric assessment. One of the most often applied assessment tests regarding mobility is the Timed Up \& Go (TUG) test [3]. Although assessment tests are widely used in daily clinical practice some problems have been found over time (section II). These problems impose the demand for two innovations: (1) The technical support of assessment tests in professional environments in order to make their execution more objective and less time-intensive. (2) To bring assessment tests to the domestic domain of people in order to estimate their real performance over longer periods in unsupervised situations. Recently, we have presented aTUG, a new approach to supporting the TUG assessment test by exclusive use of ambient sensor technologies [4]. The approach includes a technical apparatus which is designed to effectively support the execution of TUG in professional care facilities. On a long term, the concept behind aTUG also aims at supporting the execution of unsupervised TUG in domestic environments.

Within this paper we present the aTUG concept and its implementation of performing unsupervised mobility assessment tests, especially the TUG, in domestic environments. The use of mobility assessment tests is briefly motivated and the state of the art in technical systems for performing assessment tests and mobility analysis is described. The presented approach was evaluated in a field trial in Oldenburg, Germany. Results of the conducted field trial are presented and problems in performing unsupervised assessment tests are discussed.

\section{Medical Motivation}

Mobility is a fundamental requirement for an independent lifestyle and impairments in mobility are closely related to an increased need of care. Although mobility changes while aging, pathological reasons lead to significantly more sever changes in mobility [5]. Therefore, significant long-time changes in mobility may point to pathological causes and may thus be utilized for early diagnosis in various neurological diseases [6] and for detecting increased need of care or risk of falling [2]. However, for prevention and for ensuring a selfdependent lifestyle it is more important to detect abnormal changes in mobility than making an exact diagnosis at first. Since many elderly people suffer from multi-morbidity a diagnosis is often even hard to make. Therefore, geriatric assessment tests are designed to detect abnormal changes in certain domains but do not aim at supporting a detailed diagnosis. The TUG test [3] is the probably most frequently used mobility assessment test in the field of geriatrics. Within TUG a stopwatch is used to measure the time a patient takes to complete a set of components: rise from a chair, walk $3 \mathrm{~m}$, 
turn around, walk back, and sit down again. According to the time taken by the patient to complete the test, he or she is arranged into a result group which gives a hint to the treating caregiver for required actions.

However, TUG is most often only applied in professional care facilities after an acute incident already took place. It is not yet and no longer applied when patients are at home. Chances to identify gaps between capacity and performance as proposed within WHO's International Classification of Functioning, Disability and Health (ICF) [7] are not exploited. Additionally, despite its use in every-day clinical practice and various studies, the TUG test is limited itself. For the sake of simplicity it focuses only on the time used by the patients to complete the whole test. Time taken to perform the single components of the test and other deficits of moving are ignored. Therefore, Wall et al. [8] proposed the so called Expanded Timed Get-up-and-Go (ETGUG).

In summary, there is a clear need for technical support in performing component-based TUG supervised in professional care facilities and for implementing mobility assessments unsupervised in domestic environments in order to objectively measure capacity and performance of patients.

\section{STATE OF THE ART}

Today, gait and balance analysis is mainly executed in specialized wards of care facilities using high-tech equipment like marker-based vision systems. Many geriatric stations are still using only stop-watches to execute their mobility assessment tests. Within this section we describe research approaches to executing the TUG assessment test and to instrumented mobility analysis in domestic environments and limitations of those approaches.

\section{A. Technical TUG}

Instrumented TUG has so far only been implemented using body-worn inertial sensors. TUG-T [9] is a technicallysupported version of TUG by use of two inertial sensors that measures the duration of six components (standing-up, walking forward, turn 1 , walking back, turn 2, sitting down). Therapists found a good correlation $(r=0.998)$ between the total time measured by TUG-T compared to a video analysis of 20 hemiplegic patients.

More sophisticated approaches measure parameter of gait and balance while performing the TUG test. Greene et al. [10] employed two inertial sensors to assess the gait and balance of 349 community-dwelling elderly during TUG. Overall, 29 from 44 computed features provided significant discrimination between patients with a fall history and those without. Weiss et al. [11] found that their instrumented TUG by use of a single accelerometer can be used to discriminate 23 fallers from 18 health control subjects while the stopwatch-based version can not. Marschollek et al. examined a group of 110 geriatric inpatients, 26 fallers, 84 non-fallers, using a 3D accelerometer attached to a waist belt [12]. Using only assessment data to distinguish fallers from non-fallers yielded a sensitivity of $38.5 \%$ and a specificity of $97.6 \%$, including sensory data as well increased those values to $57.7 \%$ and $100 \%$ respectively. Recently, the proposed system was extended with a monocular camera system in order to enhance computation of gait parameters and to enable recognition of TUG components [13]. Salarin and Zampieri developed iTUG an instrumented version of TUG utilizing seven inertial sensors [14] that measures duration of four components (sit-to-stand, steadystate gait, turning, turn-to-sit) and a set of balance and gait parameters. iTUG was utilized to perform mobility assessment of Parkinson's Disease patients in inpatient situations and at home. Significant differences between early PD patients and age-matched control groups where found in all components of iTUG except sit-to-stand.

Recent research investigated means for execution of TUG in unsupervised situations and domestic environments. Narayanan et al. [15] employ a waist-mounted 3D accelerometer within their so-called Directed Routine (DR). DR is a set of movements, including TUG, which are meant to be performed in a controlled manner but unsupervised by the elderly themselves in order to estimate risk of falling. Within a preliminary, supervised examination of DR with 68 elderly patients 54 features were computed from the sensor recordings. However, all 9 features computed from TUG were found to be not discriminative between fallers and non-fallers. Regarding an unsupervised application in domestic environment the authors state that the most limiting constraint will be the possible misplacement of the accelerometer device by patients.

\section{B. Mobility Tele-Monitoring}

Two main approaches arose for mobility tele-monitoring using either wearable sensors or sensors installed into the environment. Such environments are also called Health Smart Homes. A good survey of systems employing body-worm sensors can be found in [16]. Within this section we focus on ambient approaches with a special regard to monitoring elderly or demented people. Pavel and Cameron [17] employ passive motion sensors placed in various rooms of a flat. Gait velocity computed by dividing known distances between rooms by measured transition times yielded rather imprecise results. By placing three motion sensors along a frequently used walking path within a flat much more precise results could be obtained. Recently, the use of laser range scanners (LRS) for gait analysis has been investigated. Palleja et al. [18] have presented an approach to basic gait analysis. By measuring the distance to patients' legs while walking along a straight line towards the used LRS walking speed and some additional parameters like average step width or swing and stance phase time are computed. Within our own work [19] we have demonstrated the use of a LRS for precise assessment of self-selected gait velocity in domestic environments. The approach continuously tracks a moving person's center of mass and computes his or her gait velocity even when changing walking direction or standing still in between. The approach does not limit the peoples' walking path but is not capable of tracking the patients' feet separately.

Marker-less optical tracking for gait analysis has been inves- 
tigated using the Microsoft Kinect sensor. Stone and Skubic [20] have developed an approach using two Kinect sensors which they compared to a previously developed web-camerabased system and a Vicon marker-based optical system. Good correlations were found during 18 walking trials. Regarding deployment of the Kinect-based approach in domestic environments the authors state limitations regarding clothing not reflecting light in the infrared spectrum and for measurements in which persons move too close to walls or furniture.

\section{Limitations}

In every-day clinical practice, the TUG assessment test can be instrumented by using body-worn sensors. Regarding an application of TUG in domestic environments, body-worn sensors would have to be handled unsupervised by layman which may pose problems regarding correct placement and comfort especially when working with very old or demented people. Explicitly donning body-worn sensors may also remind people of being tested. Especially technical problems may be the reason why the DR presented by Narayanan et al. [15] remains the only approach to unsupervised assessment tests. Various ambient sensors have been employed for telemonitoring achieving different precision in assessing mobility. To the knowledge of the authors no results from unsupervised TUG assessment tests have been presented so far, neither using body-worn nor ambient sensors.

\section{APPROACH}

We present a novel approach to performing unsupervised assessment tests in domestic environments. The focus is on assessing mobility but the approach may also be applied to assessment tests from other domains. The approach builds upon the general idea to segment assessment tests into components made up of basic movement patterns. During unsupervised assessment tests the single patterns are measured independently from each other during normal movements every day and are recombined to complete assessment tests later on. Ambient sensors are used to record movements and a technical description formalizing which patterns comprise an assessment tests and their temporal and spatial constraints is used for the recombination. In order to find out which recordings represent valid assessment components we define objective quality criteria. Such criteria are formulas generating scores for all recordings representing their eligibility to measure the patient's performance regarding an assessment component.

The presented approach clearly differs from previously presented work in which researchers try to transfer the static application flow of the clinical assessment tests to the domestic domain. Supervision by a physician is often only replaced by making the patients themselves ensure adherence to the tests' textual descriptions. Such approaches undo the possible advantages of domestic assessments since patients do again perform a test while context factors are disregarded again. Using our approach, nearly all movements of a patient during every-day life are candidates for evaluating his or her performance. Even if many movements are filtered out due to insufficient data quality much more data should be available than from single test situations. By this we also hope to get a deeper insight into the open question of how long mobility has to be monitored until sever changes in patients' health status can be detected. The presented approach is part of the aTUG concept (section IV-A) to enable unsupervised assessments. The most important part of our approach is the definition and evaluation of quality criteria. Within the next sections we first briefly described the previously presented aTUG approach and formalize a set of quality criteria for the TUG assessment test. The remainder of the paper focuses on presenting and discussing the results of a conducted field trial in which the presented quality criteria have been evaluated in a domestic environment.

\section{A. The aTUG Concept For Instrumented Assessment Tests}

The ultimate aim of the aTUG concept and approach presented in [4] is to objectify and automate the execution of the component-based Timed Up \& Go assessment test. Instrumentation is done by exclusive use of ambient sensor technologies i.e. light barriers, force sensors, and a laser range scanner (LRS) which are integrated into a single apparatus i.e. a chair for easing clinical use. The concept have three expansion stages: support for (1) traditional TUG, (2) component-based TUG, and (3) mobility analysis. All stages will be implemented in professional facilities and in domestic environments, on a long term. The general concept, sensor processing techniques for component detection, and initial results have been presented previously.

\section{B. Technical Description of Assessment Tests}

Within aTUG mobility assessment tests are segmented into components which are mapped to basic movement patterns. The difference in terms is that components are the formal parts of an assessment test while the movement patterns are the concrete manifestation of components during natural movements. For TUG a segmentation has already been proposed by Wall et al. [8]. aTUG's segmentation is additionally mapped to components from the ICF [7]. Such segmentation may also be performed for other mobility assessment tests like the TinettiTest or the Chair-Rising-Test. The segmentation of assessment tests and the flow of segments comprising a test is technically formalized as finite state machines within the aTUG approach. For most supervised clinical assessment tests the segments or movement patterns have to happen according to certain temporal and spatial constraints. When it comes to unsupervised assessment tests in domestic environments the static flow of supervised clinical assessments is no longer practical. Therefore, our approach is to measure the single movement patterns comprising an assessment tests independently throughout the day without performing an explicit test. The technical assessment description is used later on to recombine the single patterns. However, not all sensor recordings from the domestic domain will contain valid data to estimate a patient's performance for every component of the assessment test. Therefore, criteria are required to evaluate recordings for 
their eligibility for every component. We call these criteria "assessment quality criteria".

\section{Assessment Quality Criteria for Timed Up \& Go}

The purpose of assessment quality criteria is to analyze if certain sensor measurements contain sufficient data to assess a certain assessment component, they do not provide any assessment results. A score is assigned to measurements according to their eligibility for the corresponding component. Which component may be contained in recorded measurements and thus which criteria are applied is detected by generated events according to our approach described in [4]. The criteria definitions are independent on any special sensors.

While the quality criteria are mainly designed for unsupervised assessment tests they may also be used for computing the quality of supervised tests in professional care facilities and thus for ensuring adherence to certain guidelines for ensuring comparability of results.

The maximum validity score $Q_{c}$ a measurement can reach is 1. For now, all criteria for a component are considered to evenly influence the validity. Therefore, all single criteria score functions $f_{\text {component }}^{\text {criterion }}(x)$ are defined as binary functions and are normalized to 1 . Some score functions do additionally tell how good a measurement can fulfill the corresponding criteria others can only tell whether it is fulfilled or not. All criteria scores of a component's quality function are summed up and divided by the number of criteria \# criteri $_{\text {component }}$ of a component to find the overall validity score. The general validity formula 1 is:

$$
Q_{c}=\frac{\sum_{c=1}^{\# \text { criteria }}{ }_{\text {component }} f_{\text {component }}^{\text {criterion }}(x)}{\# \text { criteria }}
$$

Since during the conducted field trial we were only able to evaluate the quality criteria for walking there/back and turning we will only describe those quality criteria within this section. Quality criteria for standing-up and sitting-down will be subject to future studies.

1) Quality Criteria for Walking There and Walking Back: Validity criteria for the components walking there and walking back include: a walking distance $s$ larger than a minimum distance $s_{\text {min }}^{\text {malking }}(2)$, a deviation in step lengths $s l$ lower than a defined threshold $s l_{\max }$ (3) and an average walking speed $v$ above a threshold $v_{\text {min }}$ in order to ensure continuous movement (4), a number of detected steps st above a minimum number of steps $s t_{\text {min }}$ in order to exclude unnatural gait patterns (5), and a measurement for unstraightness of walking str not exceeding a maximum threshold $s t r_{\max }$ in order to exclude walking around corners (6). Straightness is measured by computing the standard deviation in lengths of the difference vectors from the center of gravity of the person during each step and a theoretical ideal walking path. This path is the vector pointing from the center of the first step to the last step. These criteria are defined in formulas 2 to 6 :

$$
f_{\text {walking }}^{\text {distance }}(x)=\left\{\begin{array}{l|l}
1 & x>s_{\text {min }}^{\text {walking }} \\
\frac{x}{s_{\text {min }}^{\text {walking }}} & \text { otherwise }
\end{array}\right.
$$

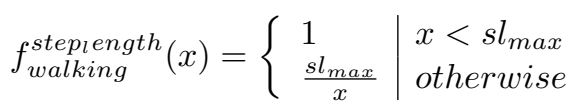

$$
\begin{aligned}
& f_{\text {walking }}^{\text {speed }}(x)=\left\{\begin{array}{l|l}
1 & x>v_{\text {min }} \\
\frac{x}{v_{\text {min }}} & \text { otherwise }
\end{array}\right. \\
& f_{\text {walking }}^{\text {steps }}(x)=\left\{\begin{array}{l|l}
1 & x>\text { st } \\
\frac{x}{\text { stmin }} & \text { otherwise }
\end{array}\right. \\
& f_{\text {walking }}^{\text {straight }}(x)=\left\{\begin{array}{l|l}
1 & x<\text { str } \max \\
\frac{s_{\text {tr }} \max }{x} & \begin{array}{l}
\text { otherwise } \\
\text { ther }
\end{array}
\end{array}\right.
\end{aligned}
$$

2) Quality Criteria for Turning: Turning has three quality criteria: The first criterion defines that the angle of the turning $\alpha$ should be above a certain threshold $\alpha_{\min }$ and below a threshold $\alpha_{\max } . \alpha$ is the angle computed between two vectors: The first vectors points from the center of first step during the turning to the point with the maximum distance from both the first and the last step of the turning. The second vector points from the maximum distance point to the center of the last step. There should be no forward movement i.e. the distance walked $s$ should not exceed a certain threshold $s_{\max }^{\text {turning }}$. Additionally, in order to separate turning from standing, the duration of the turning $t_{\text {turning }}$ between the first and the last step of the turning should not exceed a duration threshold $t_{\text {max }}^{\text {turning }}$. These criteria are defined in formulas 7 and 9:

$$
\begin{aligned}
& f_{\text {turning }}^{\text {angle }}(x)=\left\{\begin{array}{l|l}
1 & \alpha_{\min }<x<\alpha_{\max } \\
0 & \text { otherwise }
\end{array}\right. \\
& f_{\text {turning }}^{\text {distance }}(x)=\left\{\begin{array}{l|l}
1 & x<s_{\text {max }}^{\text {turning }} \\
\frac{s_{\text {max }}^{\text {turning }}}{x} & \text { otherwise }
\end{array}\right. \\
& f_{\text {turning }}^{\text {duration }}(x)=\left\{\begin{array}{l|l}
1 & x<t_{\text {max }}^{\text {turning }} \\
\frac{t_{\text {max }}^{\text {turning }}}{x} & \text { otherwise }
\end{array}\right.
\end{aligned}
$$

\section{EXPERIMENT}

An experiment conducted during a field trial had two main aims: (1) to proof the general feasibility of quality criteria to examine sensor recordings of a laser range scanner (LRS) for the components walking there/back and turning and (2) to compare gait velocity computations from a supervised TUG test to gait velocity results computed from LRS measurements and consecutive activations of home automation sensors (approach presented in [19]) during unsupervised assessments.

\section{A. Methods}

The field trial was conducted over a period of five weeks, from $2011 / 10 / 10$ to $2011 / 11 / 10$. Five community-dwelling elderly people aged 64-84 years $(2 \mathrm{~m}, 3 \mathrm{f})$ living alone and mostly independent participated but only the data of a single flat is evaluated during this experiment. Home automation (HA) sensors, five light barriers and five reed contacts, were installed in all flats. Figure 1 shows an abstracted room model of a flat including sensor placement (grey boxes, LB=light barrier, $\mathrm{RC}=$ reed contact) and computed walking paths (lines) at the left side. The model is used to compute the length of 


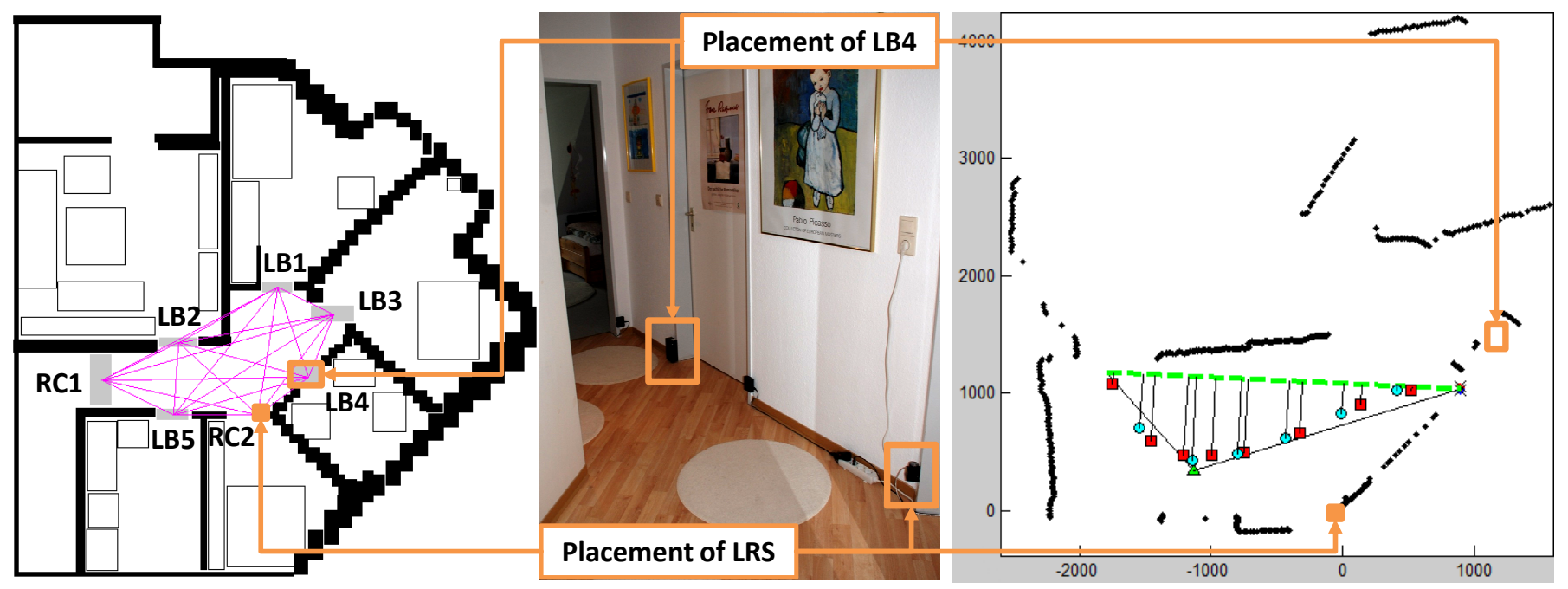

Fig. 1. Abstracted Room Model, Sensor Placement, and Laser Range Scanner Recordings During Field Trial

possible moving paths between those sensors (details in [21]). Only the flat of a 76 years old female participant was equipped with a LRS (Hokuyo URG-04LX) which was placed at a central point within the floor (Figure 1 in the middle). The sensor recorded continuously and each activation of a HA sensor was marked within the data stream. Figure 1 shows a visualization of LRS in a Cartesian coordinate system at the right. Black dots indicate measured environment. Circles and squares indicate stand phases for the left and right leg recognized from someone walking along the floor. The bold dashed line indicates the ideal walking path for this measurement to which differences for each stand phase are computed in order to compute the straightness of walking. As a clinically validated reference value each participant completed six Timed Up \& Go assessment tests using the aTUG apparatus before and four tests after the field trial in an unobstructed room.

Over a period of five weeks a total amount of 189 GB sensor recordings were collected. These data included overall 105050 activations of HA sensors from which 27595 traversed walking paths could be detected. 170 different walking paths were defined in all flats together. However, for the purpose of this evaluation, only data recorded in the fully-equipped flat are considered. In this flat, 8766 walking path transitions could be measured by HA sensors and LRS continuously. Before evaluating, recordings longer than 60 seconds and shorter than 2 seconds were excluded due to plausibility. Recordings outside those borders do in most cases either represent no clear movement like e.g. using a vacuum cleaner in the floor or do not provide enough data for analysis. The filtering leaves 2122 recordings for evaluation. For those recordings the duration for the transitions as measured by the HA sensors and the LRS and the resulting gait velocities are computed. Using only the data recorded by the LRS the real distance traversed and the quality criteria scores for walking there/back and for turning are computed.
TABLE I

TUG RESUlTs (IN S) USING A STOPWATCH (SW) AND ATUG APPARATUS

\begin{tabular}{|c|l|l|l|l|l|l|l|}
\hline & SW & \multicolumn{2}{|l|}{ aTUG } & \multicolumn{2}{l|}{} \\
\hline$\#$ & Total & Total & $\begin{array}{l}\text { Standing } \\
\text { Up }\end{array}$ & $\begin{array}{l}\text { Walking } \\
\text { There }\end{array}$ & Turning & $\begin{array}{l}\text { Walking } \\
\text { Back }\end{array}$ & $\begin{array}{l}\text { Sitting } \\
\text { Down }\end{array}$ \\
\hline \hline 1 & 11.50 & 9.34 & 0.46 & 3.53 & 1.04 & 2.39 & 1.92 \\
\hline 2 & 9.50 & 8.27 & 0.39 & 3.18 & 0.40 & 2.58 & 1.71 \\
\hline 3 & 8.01 & 7.41 & 0.47 & 2.19 & 1.69 & 1.89 & 1.17 \\
\hline 4 & 8.23 & 7.32 & 0.35 & 2.19 & 1.79 & 1.69 & 1.31 \\
\hline 5 & 9.80 & 8.51 & 0.58 & 3.18 & 1.19 & 1.89 & 1.68 \\
\hline 6 & 9.80 & 7.68 & 0.48 & 3.17 & 0.20 & 2.59 & 1.24 \\
\hline \hline $\bar{x}$, & 9.47, & 8.09, & 0.46, & 2.91, & 1.05, & 2.17, & 1.50, \\
$\sigma$ & 1.27 & 0.77 & 0.08 & 0.57 & 0.65 & 0.40 & 0.30 \\
\hline
\end{tabular}

\section{B. Results}

Since a clinically validated reference system for measuring gait was not available within the flats the only chance to obtain values for comparing the results of the experiment to was to perform an assessment before and after the trial was started. Table I shows the results of six TUG tests measured using a stopwatch (SW) and the aTUG apparatus. The first two tests were performed the day the experiment started, tests 3-4 at the last day of the trial, and tests 5-6 one week after the field trial was stopped. The tests were performed and evaluated exactly the same way as described in [4]. Results show that the participant completed all TUG tests within the range of $8.2 \mathrm{~s}$ to $10.74 \mathrm{~s}$ measured with SW (mean $+/-$ std), and between $7.3 \mathrm{~s}$ and $8.86 \mathrm{~s}$ measured with the aTUG apparatus. The difference of $1.38 \mathrm{~s}$ between SW measurements and measurements from aTUG are not relevant for a different classification. No significant difference between the tests performed before and after the field trial were found. Standard deviation for aTUG results is lower than for the SW measurements which points to subjective usage of the SW by the human tester. Using only the time for completing walking there and walking back, so excluding all delay from standing 
TABLE II

COMPUTED GAit Velocity VAlues (M/S) AND QUALITY CRITERIA

\begin{tabular}{|c|c|c|c|c|c|}
\hline $\begin{array}{l}\# \\
\text { S1,S2 }\end{array}$ & $\begin{array}{l}f_{\text {walking }}^{\text {speed }}, \\
(v)\end{array}$ & $\begin{array}{l}f_{\text {walking }}^{\text {distance }}, \\
(s)\end{array}$ & $\begin{array}{l}f_{\text {walking }}^{\text {step } \text { ength }}, \\
(\bar{s} l / \sigma(s l))\end{array}$ & $\begin{array}{l}f_{\text {walking }}^{\text {steps }} \\
(\text { st })\end{array}$ & $\begin{array}{l}f_{\text {walking }}^{\text {straight }}, \\
(\text { str })\end{array}$ \\
\hline $\begin{array}{l}1 \\
\mathrm{LB} 1, \mathrm{LB} 2\end{array}$ & $\begin{array}{l}0.21 \\
(0.10)\end{array}$ & $\begin{array}{l}0.97 \\
(1.94)\end{array}$ & $\begin{array}{l}0.75 \\
(0.37,0.20)\end{array}$ & $\begin{array}{l}1.00 \\
(25.00)\end{array}$ & $\begin{array}{l}1.00 \\
(0.13)\end{array}$ \\
\hline $\begin{array}{l}2 \\
\mathrm{LB} 5, \mathrm{LB} 2\end{array}$ & $\begin{array}{l}0.93 \\
(0.47)\end{array}$ & $\begin{array}{l}0.51 \\
(1.02)\end{array}$ & $\begin{array}{l}1.00 \\
(0.40,0.10)\end{array}$ & $\begin{array}{l}1.00 \\
(6.00)\end{array}$ & $\begin{array}{l}1.00 \\
(0.06)\end{array}$ \\
\hline$\ldots$ & $\ldots$ & $\ldots$ & $\cdots$ & $\cdots$ & $\cdots$ \\
\hline $\begin{array}{l}2030 \\
\text { LB2,RC2 }\end{array}$ & $\begin{array}{l}1.00 \\
(0.62)\end{array}$ & $\begin{array}{l}0.89 \\
(1.78)\end{array}$ & $\begin{array}{l}1.00 \\
(0.60,0.04)\end{array}$ & $\begin{array}{l}1.00 \\
(6.00)\end{array}$ & $\begin{array}{l}1.00 \\
(0.02)\end{array}$ \\
\hline$\overline{\bar{x}, \sigma}$ & $\begin{array}{l}0.84,0.26 \\
(0.53,0.22)\end{array}$ & $\begin{array}{l}\mathbf{0 . 8 5 , 0 . 2 3} \\
(2.33,1.00)\end{array}$ & $\begin{array}{l}0.93,0.15 \\
(0.47,0.11 / \\
0.13,0.09)\end{array}$ & $\begin{array}{l}0.97,0.12 \\
(11.72,5.95)\end{array}$ & $\begin{array}{l}0.76,0.29 \\
(0.22,0.20)\end{array}$ \\
\hline $\begin{array}{l}\bar{x}, \sigma \quad: \\
Q_{c}>0.5\end{array}$ & $\begin{array}{l}0.84,0.26 \\
(0.53,0.22)\end{array}$ & $\begin{array}{l}0.85,0.23 \\
(2.33,1.00)\end{array}$ & $\begin{array}{l}0.93,0.15 \\
(0.47,0.11 / \\
0.13,0.09)\end{array}$ & $\begin{array}{l}0.97,0.12 \\
(11.73,5.95)\end{array}$ & $\begin{array}{l}0.76,0.29 \\
(0.22,0.20)\end{array}$ \\
\hline $\begin{array}{lc}\bar{x}, \sigma & : \\
Q_{c}>0.75\end{array}$ & $\begin{array}{l}0.90,0.19 \\
(0.58,0.19)\end{array}$ & $\begin{array}{l}.89,0.19 \\
(2.46,0.92)\end{array}$ & $\begin{array}{l}\mathbf{0 . 9 6 , 0 . 1 1} \\
(\mathbf{0 . 4 7 , 0 . 1 0} / \\
\mathbf{0 . 1 2 , 0 . 0 8 )}\end{array}$ & $\begin{array}{l}\mathbf{0 . 9 9 , 0 . 0 6} \\
(11.48,4.97)\end{array}$ & $\begin{array}{l}0.77,0.29 \\
(0.21,0.19)\end{array}$ \\
\hline $\begin{array}{l}\bar{x}, \sigma: \\
Q_{c}=1\end{array}$ & $\begin{array}{l}1.00,0.01 \\
(0.71,0.12)\end{array}$ & $\begin{array}{l}1.00,0.00 \\
(2.76,0.47)\end{array}$ & $\begin{array}{l}1.00,0.00 \\
(0.47,0.04 / \\
\mathbf{0 . 0 9 , 0 . 0 3 )}\end{array}$ & $\begin{array}{l}1.00,0.00 \\
(11.54,2.53)\end{array}$ & $\begin{array}{l}1.00,0.01 \\
(0.09,0.04)\end{array}$ \\
\hline
\end{tabular}

(a) Walking There/Back

\begin{tabular}{|c|c|c|c|c|}
\hline $\begin{array}{l}\# \\
\text { S1,S2 }\end{array}$ & $v$ & $\begin{array}{l}f_{\text {turningle }}^{\text {ang }}, \\
(\alpha)\end{array}$ & $\begin{array}{l}f_{\text {turninge }}^{\text {distance }} \\
(s)\end{array}$ & $\begin{array}{l}f_{\text {turning }}^{\text {duration }} \\
(t)\end{array}$ \\
\hline $\begin{array}{l}1 \\
\text { LB2,LB4 }\end{array}$ & 0.60 & $\begin{array}{l}1.00 \\
(27.12)\end{array}$ & $\begin{array}{l}0.56 \\
(2.67)\end{array}$ & $\begin{array}{l}0.67 \\
(4.48)\end{array}$ \\
\hline $\begin{array}{l}2 \\
\text { LB4,LB3 }\end{array}$ & 0.09 & $\begin{array}{l}1.00 \\
(47.73)\end{array}$ & $\begin{array}{l}1.00 \\
(0.60)\end{array}$ & $\begin{array}{l}0.46 \\
(6.48)\end{array}$ \\
\hline$\cdots$ & $\cdots$ & $\cdots$ & $\cdots$ & $\cdots$ \\
\hline $\begin{array}{l}91 \\
\mathrm{RC} 2, \mathrm{LB} 5\end{array}$ & 0.06 & $\begin{array}{l}1.00 \\
(45.59)\end{array}$ & $\begin{array}{l}1.00 \\
(0.71)\end{array}$ & $\begin{array}{l}0.24 \\
(12.75)\end{array}$ \\
\hline $\begin{array}{l}92 \\
\text { LB4,LB3 }\end{array}$ & 0.40 & $\begin{array}{l}1.00 \\
(36.15)\end{array}$ & $\begin{array}{l}1.00 \\
(0.16)\end{array}$ & $\begin{array}{l}1.00 \\
(0.40)\end{array}$ \\
\hline$\overline{\bar{x}}, \sigma$ & $\begin{array}{l}0.22 \\
0.15\end{array}$ & $\begin{array}{lr}.00, & 0.00 \\
(35.91, & 17.19)\end{array}$ & $\begin{array}{l}0.81,0.21 \\
(1.83,0.90)\end{array}$ & $\begin{array}{lr}0.39, & 0.28 \\
(12.23, & 7.61)\end{array}$ \\
\hline \begin{tabular}{|lr}
$\bar{x}, \sigma$ & $:$ \\
$Q_{c}>0.5$
\end{tabular} & $\begin{array}{l}0.22 \\
0.15\end{array}$ & $\begin{array}{lr}1.00, & 0.00 \\
(35.91, & 17.19)\end{array}$ & $\begin{array}{l}0.81,0.21 \\
(1.83,0.90)\end{array}$ & $\begin{array}{lr}0.39, & 0.28 \\
(12.23, & 7.61)\end{array}$ \\
\hline $\begin{array}{lc}\bar{x}, \sigma & : \\
Q_{c}> & 0.75\end{array}$ & $\begin{array}{l}0.27 \\
0.14\end{array}$ & $\begin{array}{lr}1.00, & 0.00 \\
(34.49, & 18.75)\end{array}$ & $\begin{array}{l}0.97, \quad 0.07 \\
(1.16,0.47)\end{array}$ & $\begin{array}{lr}0.65, & 0.27 \\
(5.27, & 2.47)\end{array}$ \\
\hline $\begin{array}{l}\bar{x}, \sigma: \\
Q_{c}=1\end{array}$ & $\begin{array}{l}0.36 \\
0.10\end{array}$ & $\begin{array}{lr}1.00, & 0.00 \\
(37.58, & 16.02)\end{array}$ & $\begin{array}{l}1.00,0.00 \\
(0.77,0.39)\end{array}$ & $\begin{array}{ll}1.00, & 0.00 \\
(2.22, & 0.96)\end{array}$ \\
\hline
\end{tabular}

(b) Turning up, sitting down, or turning, and the total walking length of $6 \mathrm{~m}$ a mean gait velocity of $1.18 \mathrm{~m} / \mathrm{s}(\mathrm{min} .1 .01 \mathrm{~m} / \mathrm{s}$, max. $1.45 \mathrm{~m} / \mathrm{s}$ including standard deviations) for straight walking was computed. We use this value as a clinical reference value for capacity in walking speed during our evaluation.

Additionally, thresholds for quality criteria were computed during TUG using the aTUG apparatus. Regarding straight walking the minimum walking distance $d_{\min }^{\text {walking }}$ was set to $2 \mathrm{~m}$ since in many households no straight path of $3 \mathrm{~m}$ is available (in the equipped flat as well). The minimum walking velocity $v_{\min }$ was set to $0.85 \mathrm{~m} / \mathrm{s}$ which is mean velocity minus double standard deviation measured during the tests. The maximum deviation in step length $s l_{\max }$ was set to the double standard deviation measured, $150 \mathrm{~mm}$. Half of the number of steps detected in mean during the test, five steps, was used for $s_{\text {min }}$. The maximum distance a person should in sum differ from a straight walking line $s t_{\max }$ was $150 \mathrm{~mm}$ which ensures a rather straight walking. Again this value is the mean plus the double standard deviation measured. Regarding turning the angles $\alpha_{\min }$ and $\alpha_{\max }$ were set to be $1^{\circ}$ and $60^{\circ}$. The maximum turning distance $d_{\max }^{\text {turning }}$ was set to $1.5 \mathrm{~m}$, the maximum turning duration $t_{\max }^{\text {turning }}$ to $2.36 \mathrm{~s}$ which is the duration for turning during the tests plus the double standard deviation.

Our first aim is to proof that the defined quality criteria are suitable to evaluate measurements from the LRS according to their eligibility to measure walking there/back and turning. Table II(a) and (b) shows the computed scores and the corresponding criteria in brackets for some transitions (path as shown in Figure 1 printed in the first column) classified as walking there/back respectively for turning. Looking at the single rows one can see that scores for the single criteria decrease the more they differ from the defined thresholds. Most interesting are the computed means $\bar{x}$ and standard deviations $\sigma$ for the scores and criteria when filtering according to the total validity score as shown in the last four rows. It has to keep in mind that scores are cut at a maximum value of 1 which means that the mean scores will not grow as fast as the corresponding criteria. Overall 2030 transitions for walking were found. 2028, 1735, 382 had a validity score above 0.5 , 0.75 , and 1 . The larger the computed validity score the closer is the computed mean gait velocity $\bar{v}$ to the reference value of $1.18 \mathrm{~m} / \mathrm{s}$. Therefore we conclude that the defined quality criteria can be used to filter sensor recordings so that only those recordings are kept that represent valid results. Overall a mean walking distance $\bar{s}$ of $2.76 \mathrm{~m}$, a mean step length $\bar{s} l$ of $0.47 \mathrm{~m}$, a mean number $\bar{s} t$ of 12 steps per transition, and a mean standard deviation $s \bar{t} r$ of only $9 \mathrm{~cm}$ in straightness were found for all measurements with a score of 1 . The straightness criteria influenced the score most, even reaching only a mean score of 0.77 when filtering results with a total score less than 0.75 . The standard deviation for straightness is always high. Similar results were found for turning. Again scores decrease the less similar a movement is to turning. Overall only 92 transitions were found to represent a clear turning. From those all 92 had a score above $0.5,34$ a score larger than 0.75 , and only as little as 7 a score of 1 . This does also mean that turning did happen much more infrequently during every-day life than walking straightly - at least within the floor of this flat. The mean turning angle $\bar{\alpha}$ was 37.58 degrees. Mean distance $\bar{s}$ walked during turning was $0.77 \mathrm{~m}$, mean duration $\bar{t}$ was $2.22 \mathrm{~s}$. The computed mean gait velocity during turning was $0.22 \mathrm{~m} / \mathrm{s}$ over all measurements, respectively $0.22 \mathrm{~m} / \mathrm{s}, 0.27 \mathrm{~m} / \mathrm{s}$, and $0.36 \mathrm{~m} / \mathrm{s}$ for measurements with a total validity score above $0.5,075$, and 1 respectively. Again results come closer to the 
reference values the higher the validity score is. Regarding the influence of criteria upon the validity score the turning angle $\alpha$ does in all cases reach a score of 1 . Distance during turning reaches equally good results. Highest fluctuations and influence on the score are found for duration of turning. A reason might be that turning at home does often not happen without walking somewhere before or after and can thus only hardly be measured without walking for some distance. Such walking before and after the turning does lead to smaller scores for many measurements regarded to be turnings.

The second aim is to compare results for gait velocity computation during supervised tests and unsupervised tests using the LRS and HA sensors separately. Table III shows the computed gait velocities $v_{H A}$ using HA sensors and $v$ using the LRS for some available walking path separately. Values in brackets show corresponding standard deviations. Regarding the LRS, gait velocities have been computed from measurements with different validity scores. Additionally, the first column shows the number of total transitions available for each walking path in brackets. Only measurement classified as straight walking are included. Similar to an experiment conducted in a laboratory presented in [19] computations based on LRS measurements are more precise and come closer to the reference value of $1.18 \mathrm{~m} / \mathrm{s}$ when excluding measurements with lower scores. This does mainly result from the fact that the LRS measures the real distance walked and does not rely on previously measured theoretical distances between sensors. Additionally, the LRS is able to detect standing still in-between and does exclude the corresponding measurements from the gait velocity computation. In the mean, all results obtained from LRS measurements are more precise than those obtained from HA sensors $(0.53,0.53,0.58,0.71 \mathrm{~m} / \mathrm{s}$ vs. $0.5 \mathrm{~m} / \mathrm{s})$. Standard deviations are also lower. However, results from HA sensors are not filtered in any way. There is certainly a chance to get better results by applying statistical methods to data obtained from HA sensors. The remaining difference between the

TABLE III

Computed Gait Velocity Values (M/S) For Single Walk Paths

\begin{tabular}{|c|c|c|c|c|c|}
\hline & $\overline{\text { HA }}$ & $\overline{\text { LRS }}$ & & & \\
\hline Path & $v_{\bar{H} A}$ & $\bar{v}$ & $\begin{array}{l}\bar{v} \\
Q_{c}>0.5\end{array}$ & $\begin{array}{l}\bar{v} \text { with } Q_{c}> \\
0.75\end{array}$ & $\begin{array}{l}\bar{v} \quad \text { with } \\
Q_{c}=1\end{array}$ \\
\hline $\bar{\cdots}$ & $\cdots$ & $\cdots$ & $\ldots$ & $\ldots$ & $\cdots$ \\
\hline $\begin{array}{l}\text { LB2,LB3 } \\
\text { (266) }\end{array}$ & $\begin{array}{l}0.58 \\
(0.25)\end{array}$ & $\begin{array}{l}0.62 \\
(0.18)\end{array}$ & $0.62(0.18)$ & $0.64(0.15)$ & $0.68(0.07)$ \\
\hline $\begin{array}{l}\text { LB2,LB4 } \\
\text { (239) }\end{array}$ & $\begin{array}{l}0.50 \\
(0.18)\end{array}$ & $\begin{array}{l}0.58 \\
(0.20)\end{array}$ & $0.58(0.20)$ & $0.60(0.18)$ & $0.67(0.10)$ \\
\hline $\begin{array}{l}\text { LB2,LB5 } \\
\text { (375) }\end{array}$ & $\begin{array}{l}0.43 \\
(0.22)\end{array}$ & $\begin{array}{l}0.41 \\
(0.20)\end{array}$ & $0.41(0.20)$ & $0.48(0.15)$ & $\mathrm{NaN}$ \\
\hline $\begin{array}{l}\text { LB3,LB4 } \\
\text { (102) }\end{array}$ & $\begin{array}{l}0.25 \\
(0.09)\end{array}$ & $\begin{array}{l}0.48 \\
(0.22)\end{array}$ & $0.48(0.22)$ & $0.56(0.18)$ & $\mathrm{NaN}$ \\
\hline $\begin{array}{l}\text { LB3,LB5 } \\
(207)\end{array}$ & $\begin{array}{l}0.77 \\
(0.34)\end{array}$ & $\begin{array}{l}0.63 \\
(0.22) \\
\end{array}$ & $0.63(0.22)$ & $0.64(0.22)$ & $0.72(0.14)$ \\
\hline $\begin{array}{l}\text { LB4,LB5 } \\
\text { (298) }\end{array}$ & $\begin{array}{l}0.58 \\
(0.21) \\
\end{array}$ & $\begin{array}{l}0.61 \\
(0.22)\end{array}$ & $0.61(0.22)$ & $0.62(0.21)$ & $0.72(0.11)$ \\
\hline$\cdots$ & $\cdots$ & $\cdots$ & $\cdots$ & $\cdots$ & $\cdots$ \\
\hline $\begin{array}{l}\bar{x}, \sigma \\
(2030)\end{array}$ & $\begin{array}{l}0.50, \\
0.26\end{array}$ & $\begin{array}{l}0.53, \\
0.22\end{array}$ & $0.53,0.22$ & $0.58,0.19$ & $0.71,0.12$ \\
\hline
\end{tabular}

reference value and the LRS results may be explained by the general difference between capacity under ideal circumstances in a test situation and performance in obstructed situations e.g. due to walking not on a totally straight path. The mean error between LRS results obtained from measurements with a score of 1 and results from HA sensors is only $0.12 \mathrm{~m} / \mathrm{s}$ with a standard deviation of $0.09 \mathrm{~m} / \mathrm{s}$. Standard deviations for both measurement techniques are rather low (below $0.3 \mathrm{~m} / \mathrm{s}$ in the mean) which points to stable measurements. For some paths no results with scores of 1 could be found which is indicated by $\mathrm{NaN}$ values in the table. The affected paths are all formed by spatially close-by sensors which may have let to too short measurement times and thus to too little information to obtain good results. NaNs were excluded when computing mean values. In summary we conclude that our previous results from a laboratory setting are also valid in a domestic environment: Self-selected gait velocity can be computed unsupervised using HA sensors as well as with a LRS. LRS is more precise, HA sensors provide reasonable results while being more costeffective. In the domestic environment we found a gait velocity of $0.71 \mathrm{~m} / \mathrm{s}$ compared to $1.18 \mathrm{~m} / \mathrm{s}$ under ideal circumstances. For this participant and setting there is a difference of $0.47 \mathrm{~m} / \mathrm{s}$ between performance and capacity in gait velocity.

\section{Discussion and Future Work}

In the future we plan to extend the presented approach in order to be able to measure all components relevant to the TUG test by installing all sensors which are part of the aTUG apparatus in domestic environments. The presented experiment was mainly a technical trial in order to get a deeper insight into problems regarding unsupervised assessments and in order to proof the general feasibility of our envisioned approach. As a results of our experiment, formula 1 for computing the total validity $Q_{c}$ may have to be redesigned since the data has shown that criteria do in most cases not equally influence the quality of assessment data.

However, limitations of the field trial are obvious: Since only one participant's flat was equipped with a LRS results may not be representable. However, measurements from light barrier installations in four other flats show similar results as presented within this paper. A detailed analysis of all available HA measurements applying also additional techniques to filter available data will be published soon. Due to a missing validated gold standard for gait analysis to compare results of HA sensors and LRS to, it is hard to proof the general validity of outcomes. When asking people whether they were willing to have camera systems installed into their homes all people strongly refused. A third problem of domestic assessment tests is the encoding of results obtained over time. We are currently working on an encoding using the Clinical Document Architecture (CDA) format and relevant ICF codes.

Unsupervised assessment tests in domestic environments have the potential to provide a much deeper insight into peoples' remaining abilities. The differences and possible advantages have been formalized within the 3DLC model [22]. Despite all technical problems, it remains future work to clarify the 
relationship between results obtained from clinical assessment tests and those performed in domestic environments. A new field trial in three cities in Lower Saxony, Germany is currently under preparation. At each site 10-15 flats will be equipped with sensors to further investigate unsupervised assessments.

\section{CONCLUSION}

A new approach to performing unsupervised mobility assessments in domestic environments as part of the aTUG concept was presented. In difference to other approaches, we discard the static flow of assessment tests within the domestic domain. Instead our approach is based on the idea to segment assessment tests into recurring movement patterns which are measured independently by use of ambient sensor technologies. Corresponding measurements are evaluated according to quality criteria which compute a score of eligibility for usage of sensor data to assess a certain test component.

An experiment has been conducted within a field trial in Oldenburg, Germany with five elderly people. All flats were equipped with home automation (HA) sensors. A laser range scanner (LRS) was placed in one flat. TUG using the aTUG apparatus and a stopwatch was used as reference data. Results from the fully-equipped flat show that the presented quality criteria are suitable to select LRS measurements according to their eligibility to assess a certain test component. Additionally, we have shown that HA sensors and the LRS can be used to compute self-selected gait velocity unsupervised at home. The monitored patient moved with a mean gait velocity of $1.18 \mathrm{~m} / \mathrm{s}$ under ideal circumstances and reduced her speed to $0.71 \mathrm{~m} / \mathrm{s}$ during the field trial in an obstructed environment. A difference of $0.47 \mathrm{~m} / \mathrm{s}$ between capacity and performance in gait velocity for this patient and setting was found.

Within our future work we will use lessons learned during a new field trial. We will use additional sensors in order to cross-validate and replace the missing gold standard. Within a clinical study we are currently validating the aTUG apparatus and its sensors against a gait analysis system. An algorithm for recombining segments to complete assessment tests while keeping temporal and spatial constraints is under development.

\section{ACKNOWLEDGMENT}

Partially funded within the research project PAGE (BMBF grant 01FCO8044). The authors thank Mrs. BrinkmannGerdes from GSG Oldenburg for recruiting participants.

\section{REFERENCES}

[1] T. M. Gill, C. S. Williams, and M. E. Tinetti, "Assessing risk for the onset of functional dependence among older adults: the role of physical performance." J Am Geriatr Soc, vol. 43, no. 6, pp. 603-609, Jun 1995.

[2] M. Montero-Odasso, M. Schapira, E. R. Soriano, M. Varela, R. Kaplan, L. A. Camera, and L. M. Mayorga, "Gait velocity as a single predictor of adverse events in healthy seniors aged 75 years and older." J Gerontol A Biol Sci Med Sci, vol. 60, no. 10, pp. 1304-1309, Oct 2005.

[3] D. Podsiadlo and S. Richardson, "The timed "Up \& Go": a test of basic functional mobility for frail elderly persons." J Am Geriatr Soc, vol. 39, no. 2, pp. 142-148, Feb 1991.

[4] T. Frenken, B. Vester, M. Brell, and A. Hein, "aTUG: Fully-automated timed up and go assessment using ambient sensor technologies," in Proc. 5th Int Pervasive Computing Technologies for Healthcare (PervasiveHealth) Conf, 2011, pp. 55-62.
[5] F. J. Imms and O. G. Edholm, "Studies of gait and mobility in the elderly." Age Ageing, vol. 10, no. 3, pp. 147-156, Aug 1981.

[6] H. Stolze, S. Klebe, C. Baecker, C. Zechlin, L. Friege, S. Pohle, and G. Deuschl, "Prevalence of gait disorders in hospitalized neurological patients." Mov Disord, vol. 20, no. 1, pp. 89-94, Jan 2005.

[7] I. Classification, T. Who, and T. Icf, "WHO - International Classification of Functioning, Disability and Health (ICF)," pp. 205214, 2007. [Online]. Available: http://www.who.int/classifications/icf/en/

[8] J. C. Wall, C. Bell, S. Campbell, and J. Davis, "The Timed Get-up-andGo test revisited: measurement of the component tasks." J Rehabil Res Dev, vol. 37, no. 1, pp. 109-113, 2000.

[9] Y. Higashi, K. Yamakoshi, T. Fujimoto, M. Sekine, and T. Tamura, "Quantitative evaluation of movement using the timed up-and-go test," Engineering in Medicine and Biology Magazine, IEEE, vol. 27, no. 4, pp. $38-46,2008$.

[10] B. R. Greene, A. O. Donovan, R. Romero-Ortuno, L. Cogan, C. Ni Scanaill, and R. A. Kenny, "Quantitative Falls Risk Assessment Using the Timed Up and Go Test," IEEE Trans. Biomed. Eng., vol. 57, no. 12 , pp. 2918-2926, 2010.

[11] A. Weiss, T. Herman, M. Plotnik, M. Brozgol, N. Giladi, and J. M. Hausdorff, "An instrumented timed up and go: the added value of an accelerometer for identifying fall risk in idiopathic fallers," Physiological Measurement, vol. 32, no. 12, p. 2003, 2011.

[12] M. Marschollek, G. Nemitz, M. Gietzelt, K. Wolf, H. Meyer zu Schwabedissen, and R. Haux, "Predicting in-patient falls in a geriatric clinic," Zeitschrift fr Gerontologie und Geriatrie, vol. 42, pp. 317-322, 2009, 10.1007/s00391-009-0035-7.

[13] J. Spehr, M. Gietzelt, S. Wegel, Y. Kltzsch, S. Winkelbach, M. Marschollek, M. Gvercin, F. Wahl, R. Haux, and E. SteinhagenThiessen, "Vermessung von Gangparametern zur Sturzprdikation durch Vision- und Beschleunigungssensorik," in Demographischer Wandel Assistenzsysteme aus der Forschung in den Markt (AAL 2011), 2011.

[14] A. Salarian, F. B. Horak, C. Zampieri, P. Carlson-Kuhta, J. G. Nutt, and K. Aminian, "iTUG, a sensitive and reliable measure of mobility." IEEE Trans Neural Syst Rehabil Eng, vol. 18, no. 3, pp. 303-310, Jun 2010.

[15] M. R. Narayanan, S. J. Redmond, M. E. Scalzi, S. R. Lord, B. G. Celler, and N. H. L. Ast, "Longitudinal falls-risk estimation using triaxial accelerometry." IEEE Trans Biomed Eng, vol. 57, no. 3, pp. 534-541, Mar 2010.

[16] C. N. Scanaill, S. Carew, P. Barralon, N. Noury, D. Lyons, and G. M. Lyons, "A review of approaches to mobility telemonitoring of the elderly in their living environment." Ann Biomed Eng, vol. 34, no. 4, pp. 547563, Apr 2006.

[17] M. Pavel, T. Hayes, I. Tsay, D. Erdogmus, A. Paul, N. Larimer, H. Jimison, and J. Nutt, "Continuous Assessment of Gait Velocity in Parkinson's Disease from Unobtrusive Measurements," in Proc. 3rd International IEEE/EMBS Conference on Neural Engineering CNE '07, May 2-5, 2007, pp. 700-703.

[18] T. Pallej, M. Teixid, M. Tresanchez, and J. Palacn, "Measuring Gait Using a Ground Laser Range Sensor,” Sensors, vol. 9, no. 11, pp. 9133 9146, 2009.

[19] T. Frenken, M. Gövercin, S. Mersmann, and A. Hein, "Precise Assessment of Self-Selected Gait Velocity in Domestic Environments," in Pervasive Computing Technologies for Healthcare (PervasiveHealth). IEEE, 2010, ISBN 978-963-9799-89-9.

[20] E. E. Stone and M. Skubic, "Evaluation of an Inexpensive Depth Camera for Passive In-Home Fall Risk Assessment," in Pervasive Computing Technologies for Healthcare (PervasiveHealth), 2011 5th International Conference on, 2011.

[21] T. Frenken, E.-E. Steen, M. Brell, W. Nebel, and A. Hein, "Motion Pattern Generation and Recognition for Mobility Assessments in Domestic Environments," in Proceedings of the 1st International Living Usability Lab Workshop on AAL Latest Solutions, Trends and Applications. In conjunction with BIOSTEC 2011. SciTePress, 28-29 January 2011, pp. 3-12, ISBN 978-989-8425-39-3.

[22] A. Helmer, M. Lipprandt, T. Frenken, M. Eichelberg, and A. Hein, "3DLC: A Comprehensive Model for Personal Health Records Supporting New Types of Medical Applications," Journal of Healthcare Engineering, vol. 2, pp. 321-336, 2011, iSSN 2030-2295 DOI 10.1260/20402295.2.3.321. 